\title{
Le plaisir du texte : une expérience esthétique du lecteur
}

\section{The Pleasure of the Text: An Aesthetic Experience for the Reader}

\author{
Jawad HAZIM'
}

'Doctoral Student, Faculty of Literature and Human Sciences, IBN ZOHR University, Agadir, Morocco

ORCID: J.H. 0000-0002-1031-0761

\section{Corresponding author: \\ Jawad HAZIM, \\ NR 375 CITE LAGOUIRA LAKHYAM, Faculty \\ of Literature and Human Sciences, IBN \\ ZOHR University, Agadir, Morocco, 80000 \\ Agadir-Maroc \\ E-mail: jawadhazim@gmail.com \\ Submitted: 22.12 .2020 \\ Revision Requested: 06.04.2021 \\ Last Revision Received: 07.04.202 \\ Accepted: 07.05.2021}

Citation: Hazim, J. (2021). Le plaisir du texte : une expérience esthétique du

lecteur. Litera, 31(2), 619-632.

https://doi.org/10.26650/LITERA2020-845342

\begin{abstract}
RÉSUMÉ
La notion du plaisir a souvent été source de débats et positions aporétiques aussi bien dans les réflexions critiques que dans la philosophie de l'art. Or, évoquer la notion du plaisir dans un processus de lecture implique essentiellement de mettre en évidence la relation triadique auteur - texte - lecteur. Toutefois, le sujet/lecteur pourrait avoir une sensation du plaisir face à un texte sans pour autant penser à son auteur, la seule entité actualisable à présent demeure le texte littéraire. II s'agit de fait d'un "plaisir bien plus intellectuel» (Barthes, 1973, p. 11), ce qui inscrit celui-ci dans une dimension tant critique qu'esthétique. Si nous prenons le plaisir dans une acception euphorique, il s'agirait dès lors d'un sentiment qui relève de l'affect, alors ce dernier de ce point de vue n'est rien d'autre que la réconciliation du sensible (lecteur) et de l'intelligible du texte littéraire. Réconciliation qui passe par un regard, une attitude, face à cette «machine paresseuse» (Eco,1985, p. 34) que l'expérience esthétique actualise. Le présent travail examinera de près le rôle du sujet/lecteur dans la lecture avant d'atterrir sur les notions du plaisir, d'expérience, et la dimension esthétique qui les sous-tend. Partant du postulat que le plaisir est une expérience esthétique du lecteur, Il convient en bonne logique de s'interroger sur la notion du plaisir dans une dimension esthétique; en tant qu'expérience du lecteur, de demander dans quelle mesure le plaisir est une forme d'intentionnalité du sujet/lecteur. Ce faisant, l'étude portera sur l'examen des renvois parodiques et les écarts ironiques dans quelques passages des Frères Karamazov de Dostoïevski et La part du fils de Jean-Luc Coatalem.
\end{abstract}

Mots-clés: Plaisir, esthétique, expérience, lecture, lecteur

\section{ABSTRACT}

The notion of pleasure has often been the source of debates and aporetic positions both in critical reflections and in the philosophy of art. However, to evoke the notion of pleasure in a reading process implies essentially to highlight the triadic relation author - text - reader. However, the subject/reader could have a feeling of pleasure in front of a text without thinking about its author, the only entity that can be actualized now remains the literary text. It is in fact a «much more intellectual pleasure» (Barthes, 1973, p. 11), which places it in a critical and aesthetic dimension. If we take pleasure in a euphoric sense, it would therefore be a feeling that is related to the affect, then the latter from this point of view is nothing other than the 
reconciliation of the sensitive (reader) and the intelligible of the literary text. Reconciliation that passes through a look, an attitude, facing this «lazy machine» (Eco,1985, p. 34) that the aesthetic experience actualizes. This work will examine closely the role of the subject/reader in reading before landing on the notions of pleasure, experience, and the aesthetic dimension that underlies them. Starting from the premise that pleasure is an aesthetic experience of the reader, It is fitting i logic to question the notion of pleasure in an aesthetic dimension; as an experience of the reader, ask to what extent pleasure is a form of intentionality of the subject/reader. The study will examine the parodic references and the ironic discrepancies in some passages of Brothers Karamazov of Dostoyevsky and The part of the son of Jean-Luc Coatalem.

Keywords: Pleasure, aesthetics, experience, reading, reader

\section{EXTENDED ABSTRACT}

Reading remains one of the most complex activities in human experience. Whether it is scholarly or dilettante, the reading process is part of what we can call, as François Rastier has already described it, a semantic perception. A perception that requires the involvement of both sensory and emotional registers of the subject/reader in any form of updating a work of art and a literary work. Being the object of semiosis, construction and apprehension of meaning, the literary text also bends to this form of perception - complex of the rest - and questions another dimension that seems cardinal to us in the process of reading: the pleasure. Evoking the notion of pleasure in a reading process reveals an emotional and euphoric dimension and even seems to contradict certain particularities of the critical approach specific to the subjectivity of the subject/ reader. However, to experience pleasure in some respects comes close to making a judgment of taste. The reading of this point of view intersects the aesthetic apprehension of a work of art in general and of the literary text in particular. This is why the dimension of pleasure is added to the aesthetic component of the subject/reader and is thus part of a reading dynamic that supports both the dynamic and the reading experience. Dynamic, experience, and aesthetics may give rise to ambiguity, but the dimension of pleasure seems to combine these three notions in a formal, structural, and intentional uniqueness in the reading process. The object of this article is to irrigate critical reflections on reading activity by following the paths of the aesthetics of the text and the theories of reception. It is indeed a question of dialoguing the different theoretical postures of the reception and aesthetics of the text; of reviewing the paradigmatic crossings in a kind of phenomenology of the act of reading. In doing so, the article takes into account the active role of the subject/reader in the reception of the literary work through the textual substratum in order to show that the pleasure is not an entity intrinsically linked to the text object, but an orientation, an intentional approach, hence the qualifier of aesthetics in the experience of the subject/reader. 
In addition, to highlight the dimension of pleasure in the aesthetic experience, we pause on some passages of Brothers Karamazov of Dostoyevsky and The part of the son of Jean-Luc Coatalem. These micro-texts will serve us as an empirical object through the actualization, on the one hand of parodic references that can figure as avatar of aesthetic pleasure. Textual substrate allows more to highlight the choice of the parodic orientation waiting horizon. On the other hand, micro-texts set different horizons of expectation whose ironic differences suggest several grids of reading. The reconciliation of the different registers present in the ironic turns allows us to put the finger on the emotional, reflexive, critical, and aesthetic commitment that can in no way make the economy of the dimension of pleasure in reading. in the same view of Roland Barthes, for whom reading is a form of « much more intellectual pleasure » (Barthes, 1973, p. $11)$, we insist in this article on the fact that pleasure is also a conscious and intentional choice of the subject/reader, and part of his aesthetic experience. 


\section{Introduction}

La lecture reste l'une des activités les plus complexes dans l'expérience humaine. Qu'elle soit savante ou en dilettante, la démarche de lecture s'inscrit dans ce que nous pouvons appeler, à la suite de Rastier (Rastier, 1991, p. 121-122), une perception sémantique. Perception qui exige l'implication des registres sensoriels et affectifs du sujet/lecteur dans toute forme d'actualisation d'une œuvre d'art et à fortiori une œuvre littéraire. Étant l'objet de sémiosis, construction et appréhension du sens, le texte littéraire se plie à cette forme de perception - complexe du reste - et interroge une autre dimension qui nous parait inhérente au processus de lecture : le plaisir. Évoquer la notion du plaisir dans un processus de lecture laisse percevoir une dimension affective et euphorique de lecture et semble même contredire certaines particularités de la démarche critique propre à la subjectivité du sujet/lecteur. Or, éprouver du plaisir se rapproche à certains égards à émettre un jugement de goût et la lecture de ce point de vue recoupe l'appréhension esthétique d'une œuvre d'art en général et du texte littéraire en particulier. Raison pour laquelle la dimension du plaisir s'ajoute à la composante esthétique du sujet/lecteur et s'inscrit de ce fait dans une dynamique de lecture qui prend en charge aussi bien la dynamique que l'expérience de lecture. Dynamique, expérience et esthétique peuvent prêter le flanc à l'équivocité, mais la dimension du plaisir semble agréger ces trois notions dans une unicité formelle, structurelle et intentionnelle dans la démarche de lecture. L'objet dans cet article est de montrer que le plaisir n'est pas une entité intrinsèquement liée à l'objet texte, mais une orientation, une démarche intentionnée, d'où le qualificatif d'esthétique dans l'expérience du sujet/ lecteur.

\section{La dynamique esthétique de lecture}

Plaisir, jouissance sont des termes qui ne connaissent guère de sens univoque, de consensus quant à leur présence dans un processus de lecture. Cette dernière réclame un investissement de la part de lecteur et toute actualisation de contenu doit son achèvement pour une large part au rôle du sujet/lecteur. Avant d'entamer la question du plaisir, il convient d'emblée de mettre au jour le rôle protagoniste du sujet/lecteur. En effet, l'acte de lecture demeure l'objet de multitudes discussions théoriques. De par sa complexité, le texte littéraire implique l'engagement de sujet/lecteur afin d'en actualiser le contenu. Engagement traduit par un processus dynamique invitant le lecteur à superposer ses connaissances linguistiques et extralinguistiques en fonction 
d'itinéraires textuels offerts. La lecture est un acte généré par une dynamique entre le texte et le lecteur : le texte en tant que «pure virtualité s'il n'est pas actualisé lors de la lecture, acte par lequel il acquiert une signification » (Thérien, 2007, p. 3), il reste une entité abstraite. Il nécessite l'engagement du lecteur afin d'y conférer une signification.

Pour éclaircir et donner un peu plus de chair à cette perspective de lecture, nous pouvons dire que toute production de signification d'une œuvre d'art; et, a fortiori du texte littéraire, n'est pas une reproduction d'une donnée intrinsèque, mais il s'agit pour ainsi dire d'un processus de création;

le sens d'une œuvre n'est plus le fruit d'une explication, mais d'une action [...] I'horizon de sens est obtenu par un travail de création qui consiste à transformer la structure textuelle grâce à un processus imaginatif qui se déclenche chez le lecteur. (Gilli, Semen [en ligne] Consulté le 21 décembre 2020)

Force est donc de constater que le sujet/lecteur joue un rôle essentiel dans la production de signification, production de «l'effet esthétique» selon les termes de Wolfgang ISER. La lecture ne se cantonne pas dans un mécanisme de décodage des signes, mais une création de signifiés : le sujet/lecteur se charge de remplir les « interstices » explicites ou implicites dans la structure du texte. D'où le rôle effectif du sujet/lecteur dans la production de «l'effet esthétique». Ce faisant, le lecteur demeure le producteur de l'effet esthétique inhérent à ce mécanisme. Nous en relevons déjà les prémices de l'expérience esthétique du lecteur quant à la participation à l'actualisation de contenu.

Dans le sillage d'ISER, pour qui le texte est une entité virtuelle qui aura besoin de l'intervention du lecteur dans le processus de lecture ( la dynamique de lecture ), nous pouvons affirmer que ce produit de la sémiosis — le texte - dans la perspective d'Umberto ECO reste « une machine paresseuse qui exige du lecteur un travail coopératif acharné pour remplir les espaces de non-dit ou de déjà-dit restés en blanc» (Eco, 1985, p. 34). En outre, la lecture reste toujours un processus complexe impliquant les caractéristiques intrinsèques et extrinsèques du texte. En d'autres termes, la complexité du texte, étant donné ses structures linguistiques d'une part et extralinguistiques de l'autre, suscitent une coopération entre une entité non actualisée - le contenu - et une entité active qui en assure l'actualisation, le lecteur. La démarche active du lecteur 
l'inscrit au cœur de l'intersubjectivité — relatif au sujet — inhérente à l'expérience de lecture et lui confère un statut décisif. II n'est pas étonnant de trouver le mot «expérience» adossé à la lecture; l'idée que l'expérience est l'un des avatars de la lecture s'inscrit déjà dans la perspective théorique de Michel De Certeau; pour qui

[L]a «lecture» une activité praticable, mais également «objectivable». C'est sa capacité singulière à construire des «fictions théoriques » à partir d'une expérience «vécue» ou «lue » qui l'a mené à proposer, à la fin des années soixante-dix, cette «esquisse » à deux fronts de la lecture. (Freijomil, Les Cahiers du Centre de Recherches Historiques [En ligne] consulté le 21 décembre 2020)

Une objection pourrait se présenter à l'esprit : la lecture en tant que mode d'actualisation n'est pas une forme inavouée de jugement ou d'évaluation typiquement et purement esthétique? Bien que Dickie ( Dickie cité dans Doguet, 2007, p. 30) récuse l'idée d'une expérience esthétique en tant qu'attitude particulièrement et spécifiquement esthétique, en tant que modalité d'évaluation; cet « esthétisme» n'est en effet pour lui qu'un leurre. Mais l'esthétique de ce point de vue ne serait-il pas une perception orientée à l'endroit d'un artefact artistique — voire conceptuel? la réponse apparait clairement dans le passage de la dynamique à l'expérience esthétique du processus de lecture.

\section{De la dynamique à l'expérience esthétique}

La lecture qui prend la forme d'expérience nous incite à s'arrêter sur ce terme. En effet, l'expérience « réside dans la stimulation d'un organe sensible et dont l'objet - au sens épistémique du terme) fait par conséquent partie du monde qui nous est accessible par nos organes des sens » (Schaeffer, 2015, p. 29). Jean-Marie Schaeffer désigne l'expérience comme un ensemble de connaissances sensibles dont la source est l'ensemble des registres sensoriels situés dans l'espace et dans le temps. Toute entité n'est accessible dans ce monde que par le biais de la sensibilité de nos organes. Nous pouvons dire dès lors que l'expérience est une corrélation sensible entre d'un côté, notre perception, nos registres sensoriels; et des entités actualisées - objet de cette perception - de l'autre : Corrélation entre sens et substance pour ainsi dire. Si nous liant l'expérience dans cette perspective au processus de lecture, nous pourrions affirmer dès lors que cette phénoménologie de lecture laisse percevoir que 
[l]a réception de l'œuvre n'est pas un simple processus d'affection passive par une entité physique ou des ondes sonores, parce qu'elle est une réception qui est à l'origine d'une expérience affective intime. La production et la réception d'une œuvre d'art ne peut exister qu'au titre de communication intersubjective. (Doguet, 2007, p. 25)

Lecture et expérience affective sont en l'occurrence à l'intersection de l'orientation esthétique du lecteur. Esthétique dont l'expérience sensible et intelligible du lecteur vont l'orienter au plaisir inhérent de l'activité de lecture. Il serait peut-être hâtif d'en arriver à cette conclusion; mais l'idée que l'implication de la dimension sensible et intelligible dans le processus de lecture laisse percevoir la notion du plaisir profiler à I'horizon. En outre, parler d'expérience nécessite donc de distinguer à la suite de Doguet (Doguet, 2007, p. 30) l'expérience comme vécu subjectif (Erlebnis) et l'expérience comme relation d'interaction; comme structure logique des représentations (Erfahung). II se trouve que le processus de lecture reste une forme inavouée de l'expérience en tant que vécu subjectif (Erlebnis) et en tant que structure logique des représentations (Erfahung): l'appréhension du contenu textuel nécessite un ancrage intertextuel et expérientiel de lecture que nous pouvons qualifier d'orientation stéréotypique au même titre qu'une transposition des structures formelles et génériques de lecture. Dans une perspective sociologique, les stéréotypes «se présentent comme des clichés mentaux stables, constants et peu susceptibles de modification. Ils sont l'opinion majoritaire d'un groupe» (Mannoni, 2016, p.36). Si nous voulons donner une définition simple, loin d'être simpliste, les stéréotypes sont la somme des représentations tout en étant fixes, invariables et consensuels. Ces représentations tracent leur place dans le parcours conscient du sujet/lecteur. Ce dernier se trouve alors au cœur d'une expérience esthétique qui ne peut réduire le texte à un artefact linguistique dépourvu de toute actualisation intentionnée.

Du reste, il ne s'agit pas de pointer de doigts un raccourci entre expérience, esthétique, et la notion du plaisir; mais de souligner que l'activité de lecture est le résultat de "l'ancrage dans nos ressources cognitives et émotives de base et l'usage si particulier qui en fait - qui caractérise l'expérience esthétique» (Schaeffer, 2015, p. 29). Le plaisir s'avère donc un corolaire de l'expérience esthétique si ce n'est pas une intentionnalité inscrite dans l'objet de lecture, texte. Le qualificatif d'esthétique se justifie par le rôle actif attentif - du lecteur; de ce point de vue, l' «esthétique n'est donc pas ici "dispositionnel", mais au contraire résultatif : ce n'est l'objet [ texte] qui rend la relation esthétique, c'est 
la relation qui rend l'objet esthétique» (Genette, 1997, p. 17). Le rôle actif du lecteur lui permet de mobiliser son

attention - perception sans identification pratique - propre à l'expérience esthétique, et même sans doute un peu plus typique de notre relation esthétique aux objets naturels que de notre relation aux œuvre d'art. (Genette, 1997, p. 15)

L'esthétique trouve alors son origine aisthesis dans la mesure où l'expérience humaine et $a$ fortiori du sujet/lecteur passe par ce que Kant qualifie d' «intuition sensible » (Kant, 2015, p.112). En outre, l'expérience comme vécu subjectif (Erlebnis), ou l'expérience comme relation d'interaction; comme structure logique des représentations (Erfahung), les deux figures semblent être articulées dans le processus de lecture. Le texte littéraire objet de lecture dont «le sens est une expérience par laquelle la signification affecte et modifie-le-moi individuel d'un récepteur» (Doguet, 2007, p. 27) se plie alors à l'orientation esthétique du lecteur : responsable de son plaisir. Cette phénoménologie au sens husserlien du plaisir se heurte à la conception du « plaisir du texte [qui] peut se définir par une pratique (sans aucun risque de répression) : lieu et temps de lecture » (Barthes, 1973, p. 45). Le plaisir serait donc le cheminement syncrétique de l'orientation esthétique et l'expérience du lecteur.

\section{Plaisir entre orientation et expérience esthétique}

Pour ne citer que l'intertexte évangélique présent à titre d'exemple dans Les Frères Karamazov de Dostoïevski, celui-ci convoque un passage emprunté à l'évangile version Matthieu, XXIV, 36, indiquée dans une note, pour donner un plus de chair à la thèse soutenue de son personnage à savoir la promesse de la visite de Jésus. L'auteur semble même contribuer à l'expérience de son lecteur en plantant le décor historique et justifier son choix « L'action se passe au XVlème siècle. Tu sais qu'à cette époque il était d'usage de faire intervenir dans les poèmes les puissances célestes » (Dostoïevski, 1880, p. 655). Mais qu'en est-il du plaisir ? nous pouvons suivre Umberto Eco pour qui le plaisir est au demeurant une expérience en ce qu'elle incite le sujet/lecteur à savourer le renvoi à partir d'un « clin d'œil cultivé » (Eco, 2002, p. 171). Sauf qu'il nous parait insuffisant de placer le plaisir dans une logique purement intertextuelle ; mais de dire que le plaisir est d'assister à la tension parodique, pas assez nette qu'elle soit au départ, entre deux substrats textuels, deux univers de référence différents dans une seule et unique trame romanesque. 
C'est ainsi que Dostoïevski lève le voile sur le nihilisme nietzschéen en campant dans son récit deux personnages en total désaccord : Jésus et le grand inquisiteur. Le fils de Dieu présente bien entendu la parole divine et la promesse de retour « Je reviendrai bientôt; quant au jour et à l'heure, le Fils même ne les connaît pas, mais seulement mon Père qui est aux cieux » (Dostoïevski, 1880, p. 654). Alors que le grand inquisiteur représente la voix profane et rebelle contre la mansuétude divine tant attendue «I'humanité l'attend avec la même foi que jadis, une foi plus ardente encore, car quinze siècles ont passé depuis que le ciel a cessé de donner des gages à l'homme » (Dostoïevski, 1880, p.654). L'auteur invite même son lecteur à savourer ce plaisir, et met les premiers jalons de renvois parodiques qui se profilent à I'horizon :

Eh bien, mon petit poème eût été dans ce goût, s'il avait paru à cette époque. Dieu apparaît ; il ne dit rien et ne fait que passer. Quinze siècles se sont écoulés, depuis qu'il a promis de revenir dans son royaume. (Dostoïevski, 1880, p. 655)

L'auteur incorpore donc des citations bibliques afin d'attribuer et donner plus de crédibilité au dialogue fictif entre Jésus et le grand inquisiteur. Pour pointer du doigt la dimension du plaisir, il convient d'affirmer d'abord que l'expérience esthétique du sujet/lecteur joue un rôle important dans cet angle de vue : il faut identifier la charge parodique par l'entremise des citations bibliques ; d'où l'expérience du sujet/lecteur en matière d'encyclopédie et des textes convoqués. De plus, la perception du fragment, l'intertexte, biblique relève déjà du jugement esthétique en ce qu'il met la parole divine dans une tension avec la parole romanesque. Le plaisir pourrait dans ce cas de figure se présenter comme un cheminement syncrétique de l'orientation esthétique du texte et l'expérience du sujet/lecteur par le prisme parodique de la citation ; laquelle prend l'allure, d'une part, d'argument d'autorité pour persuader l'énonciataire et à fortiori le sujet/lecteur du caractère nihiliste de l'être , d'autre part, la citation marque le curseur sur le rôle de l'intégration des Écritures Saintes dans une trame romanesque jugée profane .Convenons-en que le travestissement parodique des Écritures Saintes reste, à certains égards, une pratique proscrite même dans la conscience réceptrice. Or, l'identification du rapport conflictuel entre le saint et le profane est déjà une perception du plaisir guidée par le schème esthétique inavoué, peut-être inavouable, qui tend à travestir les conventions établies : si l'«esthétique n'est donc pas ici "dispositionnel", mais au contraire résultatif » (Genette, 1997, p. 17), diluer les paroles divines dans une verve extatique du grand inquisiteur mène le sujet/lecteur vers une esthétique iconoclaste. 
Le plaisir en tant que relation esthétique se traduit par l'actualisation —attentive- du lecteur du travestissement parodique de la parole divine. L'élan parodique suscite le plaisir dans la mesure où il glisse une critique acerbe de la Métaphysique ambiante dans la communauté européenne au XIX siècle, notamment la société russe sous l'emprise de la religion chrétienne. Réinterroger les assises théologiques à travers une fiction romanesque procure à la fois l'enchantement du sujet/lecteur, attentif aux tours parodiques, et le désenchantement de l'idiologie dominante. L'un des frères Karamazov, Ivan, matérialiste d'esprit, dénonce la contingence du monde et la crédulité de I'homme en vue de bousculer l'emprise métaphysique répandue dans les monastères russes, "Les extrêmes se rejoignent, les contradictions vivent accouplées. Que des mystères accable l'homme » (Dostoïevski, 1880, p. 168). Un frère matérialiste d'un côté, Ivan, un moine avec une foi du charbonnier de l'autre, Aliocha, l'appartenance de ces deux frères à la famille Karamazov nous invite à se réjouir de la parade et la parodie dostoïevskienne qui ne cesse de pousser subrepticement la critique du dogmatisme jusqu'à son paroxysme.

Sans vouloir forcer le trait, nous pouvons même mettre en évidence la main invisible qui oriente le lecteur Modèle (Eco, 1985, p. 70) vers la dimension du plaisir suggérée par le texte et appréhendée par le sujet/lecteur. En effet, si nous avons emprunté à Adam Smith la notion de la main invisible, c'est dans l'espoir de lever le voile sur le rôle de l'auteur dans l'éveil du plaisir chez le sujet/lecteur. L'idée est que la perception de la tension parodique entre deux catégories de discours (Roman et Bible) , a fortiori antagonistes, n'est rien d'autre que l'actualisation signifiante de l'intention de l'auteur " sinon comme dessein, préméditation ou intention préalable, du moins comme structure, système et intention en acte » (Compagnon, 1998, p. 72) ; qui se laisse percevoir dans l'intrusion des intertextes bibliques dans les entrailles du récit dostoïevskien. L'énoncé qui prend l'allure d'un aparté «Eh bien, mon petit poème eût été dans ce goût, s'il avait paru à cette époque » (Dostoïevski, 1880, p. 655) marque un clin d'œil à l'endroit du sujet/lecteur pour saisir l'intention de l'auteur et délecter la signifiance qui se refuge dans l'écart parodique. En outre, Dostoïevski semble renverser les valeurs culturelles partagées en campant un héros, croyant et candide, mais réaliste et éveillé. Le portrait psychologique du héros dostoïevskien recèle en soi des caractéristiques antagonistes

LE LECTEUR SE figure peut-être mon héros sous les traits d'un pâle rêveur malingre et extatique. Au contraire [...] l'air pensif et calme. On m'objectera 
que des joues rouges n'empêchent pas d'être fanatique ou mystique ; or, il me semble qu'Aliocha était plus que n'importe qui réaliste (Dostoïevski, 1880, p. 52)

L'auteur a mené un travail de sape en déconstruisant la conception commune des fanatiques religieux. II s'agit en effet d'une nouvelle configuration du personnage romanesque qui révoque en doute les valeurs omniprésentes dans la société russe et en instaurant dans un même mouvement les attributs types du personnage religieux aux antipodes de la conception doxique. Le plaisir se fait sentir donc de cette manière à partir de ce contraste auctorial du portrait psychologique des hommes de Dieu. Si nous suivons Barthes, le récit dostoïevskien constitue dans la même lancée non seulement un texte de plaisir, mais il se présente comme un texte de jouissance en ce qu'il

met en état de perte, celui qui déconforte (peut-être jusqu'à certain ennui) , fait vaciller les assises historiques, culturelles, psychologiques, du lecteur , la consistance de ses gouts, de ses valeurs et de ses souvenirs, met en crise son rapport au langage. (Barthes, 1973, p.14).

Il va sans dire que le plaisir dans une relation esthétique relève du ressort de l'auteur en ce qu'il se fait sentir dans la stratégie textuelle adoptée dans la trame romanesque. La déconstruction des schèmes culturels et psychologiques ainsi que l'orientation auctoriale sont des avatars de l'intention de l'auteur qui fait office d'une esthétique délibérément définie : éveil du plaisir.

Dans le droit fil de la relation esthétique, la dimension du plaisir s'incarne par ailleurs dans l'immixtion de modes énonciatifs antagonistes, I'un sacré, l'autre profane. De ce fait, nous pouvons ajouter que le plaisir s'ajoute dans l'expérience esthétique comme relation d'interaction et structure logique des représentations (Erfahung) impliquant les trois instances : expérience, texte et auteur. Plaisir à partir duquel le sujet/lecteur «savoure les reines des formules, le renversement des origines, la désinvolture qui fait venir le texte antérieur du texte ultérieur » (Barthes, 1973, p. 32).

En outre, une autre voie peut bien nous emmener vers cette dimension du plaisir : I'ironie. La pratique de l'ironie suscite aussi bien l'expérience esthétique que l'acuité analytique du sujet/lecteur. Au sens kantien du terme, il s'agit en effet de réconcilier 
la virtuosité du sensible (perception) avec la rationalité de l'intelligible (raisonnement). Pour prendre un exemple limite, mais révélateur, dans La part du fils, Jean-Luc Coatalem nous invite avec Paol, le héros d'une enquête incessante depuis le début du roman, à explorer les arcanes de la guerre et cultive l'envie de savoir un peu plus sur les déportés des camps nazis ; sur la cause et l'origine de l'arrestation des soldats. L'enquête de Paol est en vérité une manière de dénoncer, subrepticement, avec brin d'ironie, la barbarie des camps nazie et le traitement barbare infligé aux soldats détenus :

qui, à ce moment-là , aurait pu nous apprendre que la conquête spatiale était née vingt-cinq ans plus tôt, dans les camps nazis, dont celui de Dora , où les prototypes des lanceurs avaient été mis au point, et où Paol fut prisonnier? (Coatalem, 2019, p. 165)

L'auteur/narrateur a glissé ses griffes et ses griefs par un tour ironique. La'conquête spatiale' exhibe le génie nazi dans la torture et la formation des tortionnaires. De même, I'usage de jargon technique 'prototype' aiguise l'acerbité ironique. Or, le plaisir réside bel et bien dans l'actualisation des propos grinçants sous le voile d'invention et du génie nazi. À la charge émotive s'ajoute la subtilité ironique dans la description de l'approche belliciste, à l'antipode des valeurs humaines criées sur tous les toits. Décidément, 'l'égalité et la fraternité s'exporte mal' (Coatalem, 2019, p. 94). Mieux encore, ce qu'on pourrait appeler les'vestiges de la guerre' continuent à alimenter et à irriguer la mémoire des habitants sans pour autant sidérer la conscience humaniste :

Aujourd'hui, en dépit des bombardements qui se sont succédé, le dôme colossal de béton armé de la coupole, sorte de grosse soucoupe volante de soixante-douze mètres posée en surplomb de la bourgade d'Helfaut, accueille encore le visiteur (Coatalem, 2019, p. 130)

Le narrateur semble être sidéré par la présence encore de ces pseudo-monuments. L'expérience esthétique s'incarne de ce point de vue dans tous les stéréotypes partagés (égalité, fraternité, altruisme, etc.) : un horizon d'attente marqué dans l'expérience commune : le plaisir de cette manière est « une pratique (sans aucun risque de répression): lieu et temps de lecture »(Barthes ,1973, p. 45). Raison pour laquelle le texte - lieu d'intelligibilité sémiotique - et l'investissement du sujet/lecteur - temps d'engagement sensoriel et émotionnel - concourent à l'éveil du plaisir. Qui plus est, l'hostilité des guerres et les atrocités humaines s'inscrivent en faux contre l'humanisme et les autres 
slogans enjôleurs. Le narrateur s'amuse à décrypter l'un des acronymes qui cachent tous les horreurs du siècle :

On imaginerait un prénom de femme, le diminutif de Théodora. Ou celui d'un chat, affectueux et tendre. Si certains ont affirmé qu'il s'agissait d'une fillette, celle de Fritz Sauckel, l'ordonnateur des déportations, la réalité est banale : c'est l'acronyme de deutsche organisation Reich Arbeit.Dora. (Coatalem, 2019, p. 155)

Cette description aux allures humoristiques éveille dès lors une poignée de plaisir par l'entremise du clin d'œil ironique d'autant plus que l'écart ironique convoque une grille de lecture critique à travers laquelle le sujet/lecteur « savoure l'ironie - et pas seulement le clin d'œil cultivé que lui adresse le lecteur » (Eco, 2002, p. 171). Le plaisir en tant qu'expérience esthétique engage au demeurant la contribution du sujet/lecteur dans la construction même de la fabula et secoue l'expérience — l'instar d'un vécu subjectif (Erlebnis) —, par une fibre aussi bien comique qu'ironique, afin de se rendre compte de ceux et celles qui n'ont pas la voix au chapitre. Par ailleurs, l'atmosphère morbide et mortifère exposée au fil des chapitres dans La part du fils ne permet pas de savourer l'écart entre le comique et le tragique ; or la tonalité ironique pallie l'horreur de la description et sert de grille de lecture réduisant l'écart entre l'agréable et le désagréable sous une trame autofictionnelle.

\section{Conclusion}

La dimension du plaisir reste ancrée dans l'orientation esthétique du sujet/lecteur et relative à I'horizon d'attente établi. Esthétique reste fidèle à ses racines, aisthesis au sens grec, dans la mesure où le cheminement de la lecture se structure au gré des registres sensoriels convoqués (perception, émotion, identification aux personnages, etc.) La perception des travestissements parodiques engage une connaissance, une expérience pourrait-on dire, du thème ou du texte parodié afin de jouir de ce plaisir esthétique. La lecture qui suit un itinéraire parodique acquiesce au jeu proposé par le texte et savouré par le sujet/lecteur en plaçant le plaisir dans une intentionnalité délibérément consentie par l'expérience aussi bien de l'auteur que du lecteur. En outre, relever le clin d'œil ironique inscrit la lecture dans un registre à la fois tragique et comique : Les deux registres sont deux orientations, deux horizons d'attente que la réconciliation des écarts ironiques mesure à l'aune du plaisir propre à toute expérience 
esthétique. Au rebours d'une actualisation passive de contenus textuels, la lecture en tant qu'expérience et action ponctue le processus de la sémiosis en cultivant le plaisir inhérent à l'intentionnalité, au vécu et l'expérience esthétique. II convient d'affirmer somme toute que la dimension du plaisir n'est pas une caractéristique propre à l'objet texte ou tout autre substrat sémiotique, mais elle découle de l'implication consciente et intentionnée du sujet/lecteur.

Évaluation : Évaluation anonyme par des pairs extérieurs.

Conflit d'intérêts : L'auteur n'a aucun conflit d'intérêts à déclarer.

Subvention : L'auteur n'a reçu aucun soutien financier pour ce travail.

Peer-review: Externally peer-reviewed.

Conflict of Interest: The author has no conflict of interest to declare.

Grant Support: The author declared that this study has received no financial support.

\section{Bibliographie}

Barthes, R. (1973). Le plaisir du texte. Paris : Seuil.

Coatalem, J-L. (2019). La part du fils. Paris : Stock.

Compagnon, A. (1998). Le démon de la théorie. Paris : Seuil.

Doguet, J. (2007). L'art comme communication. Paris : Armand Colin.

Dostoïevski, F. (1881). Les frères Karamazov. Domaine public : libre.

Eco, U. (1985). Lector in fabula. Paris : Grasset.

Eco, U. (2002). De la littérature. Paris : Grasset.

Freijomil, G. (2009). Les pratiques de la lecture chez Michel de Certeau. Les Cahiers du Centre de Recherches Historiques. URL: http://journals.openedition.org/ccrh/3533; DOI: 10.4000/ccrh.3533, consulté le 21/12/2020 Genette, G. (1997). L'ouuvre d'art. La relation esthétique. Paris : Seuil.

Gilli, Y. (1983). Le texte et sa lecture. Une analyse de l'acte de lire selon W. Iser. Semen. URL: http://journals. openedition.org/semen/4261, consulté le 02/02/19.

Kant, E. (2015). Critique de la faculté de juger, Trad. Alain Renaut. Paris : Flammarion.

Mannoni, P. (2016). Les représentations sociales. Paris : PUF.

Mardaga, P. (1997). Wolfgang Iser. L'acte de lecture : théorie de l'effet esthétique. URL: https://books.google. co.ma/books. Consulté le 22/12/20.

Rastier, F. (1991). Sémantique et recherches cognitives. Paris : PUF.

Schaeffer, J-M. (2015). L'expérience esthétique. Paris : Gallimard.

Thérien, G. (2007). Théorie de lecture littéraire. Québec : PUQ. 